\title{
Macular Optical Coherence Tomography Imaging in Glaucoma
}

\author{
Alireza Kamalipour, MD; Sasan Moghimi, MD \\ Hamilton Glaucoma Center, Shiley Eye Institute, Viterbi Family Department of Ophthalmology, University of California, San \\ Diego, La Jolla, CA, United States \\ ORCID: \\ Alireza Kamalipour: https://orcid.org/0000-0003-2916-9187 \\ Sasan Moghimi: https://orcid.org/0000-0002-9375-4711
}

\section{Abstract}

The advent of spectral-domain optical coherence tomography has played a transformative role in posterior segment imaging of the eye. Traditionally, images of the optic nerve head and the peripapillary area have been used to evaluate the structural changes associated with glaucoma. Recently, there is growing evidence in the literature supporting the use of macular spectral-domain optical coherence tomography as a complementary tool for clinical evaluation and research purposes in glaucoma. Containing more than $50 \%$ of retinal ganglion cells in a multilayered pattern, macula is shown to be affected even at the earliest stages of glaucomatous structural damage. Risk assessment for glaucoma progression, earlier detection of glaucomatous structural damage, monitoring of glaucoma especially in advanced cases, and glaucoma evaluation in certain ocular conditions including eyes with high myopia, positive history of disc hemorrhage, and certain optic disc phenotypes are specific domains where macular imaging yields complementary information compared to optic nerve head and peripapillary evaluation using optical coherence tomography. Moreover, the development of artificial intelligence models in data analysis has enabled a tremendous opportunity to create an integrated representation of structural and functional alterations observed in glaucoma. In this study, we aimed at providing a brief review of the main clinical applications and future potential utility of macular spectral-domain optical coherence tomography in glaucoma.

Keywords: Artificial Intelligence; Glaucoma; Imaging; Macula; Optical Coherence Tomography

J Ophthalmic Vis Res 2021; 16 (3): 478-489

\section{INTRODUCTION}

Glaucoma, characterized by progressive loss of retinal ganglion cells (RGCs) and their axons accompanied by concomitant characteristic visual field (VF) damage, is globally a leading cause

Correspondence to:

Sasan Moghimi, MD. Shiley Eye Institute, University of California, San Diego, 9500 Campus Point Drive, La Jolla, CA, 92093-0946, USA.

E-mail: sasanimii@yahoo.com

Received: 15-01-2021

Accepted: 22-04-2021

\section{Access this article online}

Website: https://knepublishing.com/index.php/JOVR

DOI: 10.18502/jovr.v16i3.9442 of irreversible blindness. ${ }^{[1-5]}$ Earlier diagnosis and monitoring of disease progression are two fundamental tasks for clinicians managing glaucoma. ${ }^{[2,6,7]}$ Developments in imaging modalities in the last three decades have led to recent advances in glaucoma diagnosis and management. ${ }^{[8-15]}$ Optical coherence tomography (OCT) is now the imaging modality of choice for

This is an open access journal, and articles are distributed under the terms of the Creative Commons Attribution-NonCommercial-ShareAlike 4.0 License, which allows others to remix, tweak, and build upon the work non-commercially, as long as appropriate credit is given and the new creations are licensed under the identical terms.

How to cite this article: Kamalipour A, Moghimi S. Macular Optical Coherence Tomography Imaging in Glaucoma. J Ophthalmic Vis Res 2021;16:478-489. 
objective assessment of glaucomatous structural alterations due to fast and highly reproducible scan acquisition. ${ }^{[16-18]}$ In clinical practice, most attentions on OCT imaging in glaucoma has been paid to the evaluation of the optic disc that together with VF assessment using $6^{\circ}$ apart test points (e.g., the 24-2 or 30-2 test pattern) constitute the common clinical paradigm. ${ }^{[19,20]}$ However, evidence shows that sole reliance on common clinical paradigm might be insufficient in certain clinical aspects. Importantly, glaucomatous damage to the macular area may happen early in the disease course and such damage can be underestimated or even missed using common clinical paradigm. ${ }^{[19]}$ Also, evaluating glaucoma progression especially at the advanced stage where optic nerve head $(\mathrm{ONH})$ and circumpapillary retinal nerve fiber layer (cpRNFL) measurements have reached to an apparent floor is limited by the current common clinical paradigm. Another challenge happens in the evaluation of glaucoma patients with high myopia where the OCT segmentation algorithms are more prone to errors at the $\mathrm{ONH}$ area as a result of anatomical alterations such as peripapillary atrophy, $\mathrm{ONH}$ tilt, and stretching of the cpRNFL. Macular OCT imaging with high resolution scans of different layers can be a useful adjunct to common clinical paradigm in these scenarios. Moreover, the application of artificial intelligence (Al) techniques to analyze the big data obtained from these high-resolution images shows promise to improve the currently available diagnostic modalities and structure-function relationships in glaucoma.

Glaucomatous damage to the macular area that contains around $50 \%$ of retinal RGCs in a multilayered fashion has been reported for a long time using histological studies. ${ }^{[18]}$ Potential damage to this area leads to impairments in the central VF which is associated with a dramatic decline on the functional status in glaucoma patients. Moreover, macular evaluation in glaucoma has recently regained a specific focus of interest based on the possibility of early involvement in the disease process. ${ }^{[21]} \mathrm{New}$ SD-OCT post acquisitional algorithms provide automated segmentation of different layers of macular area that focuses on interest in glaucoma evaluation and monitoring including macular retinal nerve fiber layer (mRNFL), ganglion cell layer (GCL), ganglion cell/inner plexiform layer (GC/IPL), and ganglion cell complex (GCC). Consequently, the utility of these SD-OCT-derived macular parameters for glaucoma detection and monitoring of disease progression have been shown in many previous studies. In this perspective, we aim at providing more insight on the potential utility of SD-OCT macular imaging in glaucoma practice.

\section{Different Macular OCT Imaging Instruments}

\section{Cirrus high-definition OCT (HD-OCT)}

Cirrus HD-OCT (Carl Zeiss Meditec, Dublin, CA) is one of the instruments that provides high macular images using SD-OCT technology [Figure 1A]. The Cirrus HD-OCT performs volumetric scan $(200 \times 200$ or $512 \times 128$ A-scans $)$ of the macula over an area of $6 \times 6 \times 2-\mathrm{mm}^{3}$ in an emmetropic eye that is centered on the fovea. In the Ganglion Cell Analysis (GCA) printout, it provides the GCIPL parameter that includes the combined thickness measurements of $\mathrm{GCL}$ and IPL. GCA printout displays global average GCIPL, minimum GCIPL, and sectoral GCIPL measurements that are presented over six wedgeshaped regions bound by a horizontally oval area $\left(4.8 \times 4.0 \mathrm{~mm}^{2}\right)$ after the removal of a central perifoveal ellipse $\left(1.2 \times 1.0 \mathrm{~mm}^{2}\right)$. In addition to the mentioned parameters, GCA provides a colorcoded deviation map of GCIPL measurements over the aforementioned elliptical area that compares localized thickness measurements to age-adjusted normative database of the built-in software. ${ }^{[18]}$

\section{RTVue SD-OCT}

The RTVue (Optovue Inc., Fremont, CA) is another SD-OCT imaging modality that is capable of performing a 3-D volumetric scan of the macula over a $7 \mathrm{~mm}$ square that is centered $0.75 \mathrm{~mm}$ temporally to the fovea. In the printout, it displays average, superior, and inferior GCC (including the combination of mRNFL, GCL, and IPL) measurements [Figure 1B]. Moreover, two colorcoded maps are provided in the software output that display the absolute GCC measurements and GCC deviation patterns based on the ageadjusted normalized database of the software. Cross-sectional high-resolution B-scans are also shown for further structural evaluation and detection of possible image artifacts. ${ }^{[18]}$ 


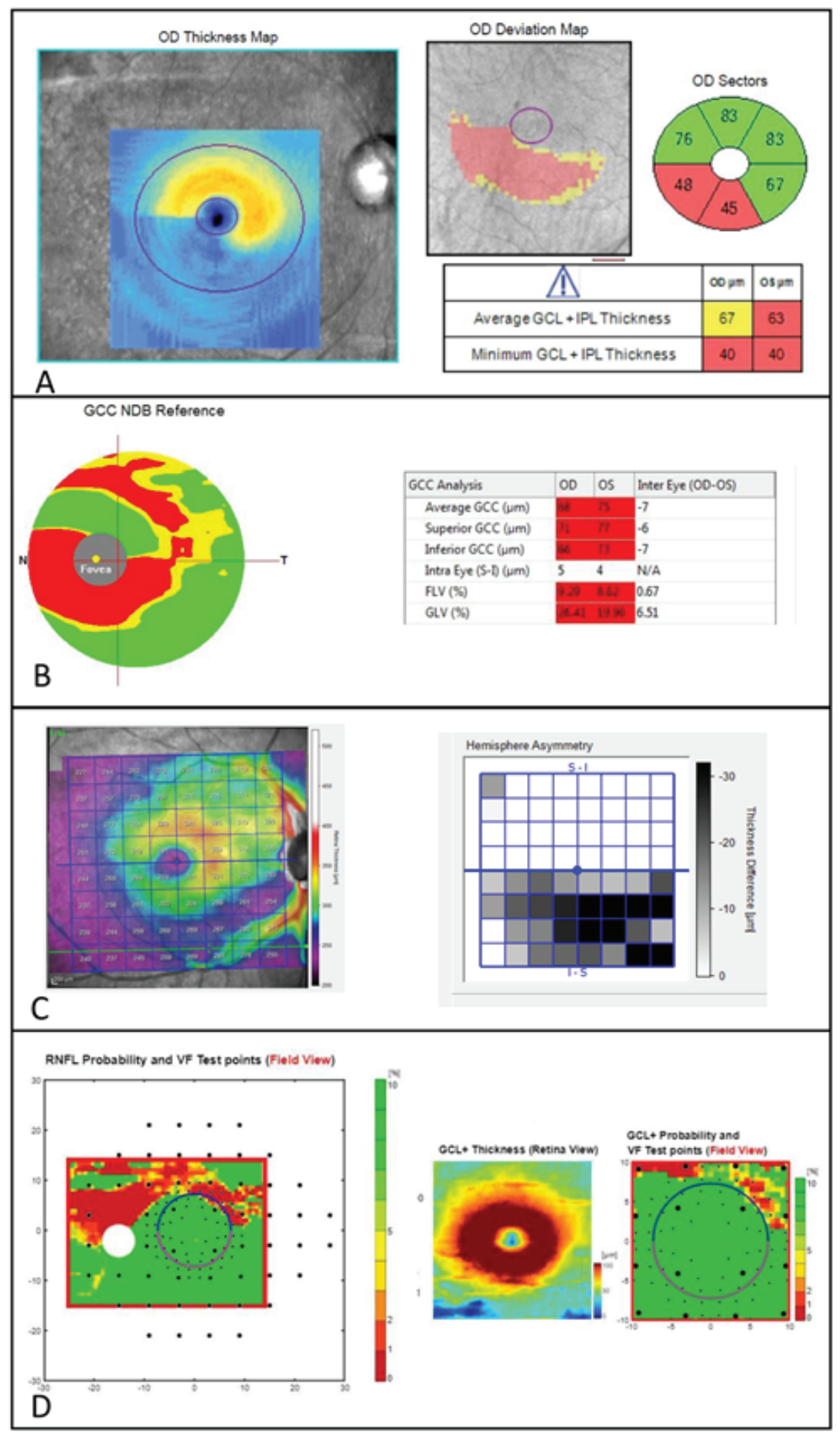

Figure 1. (A) Cirrus HD-OCT Ganglion Cell OU analysis of a patient with glaucoma. Thickness Map, Deviation, Sector Map, and average/minimum GCIPL can be provided by Cirrus OCT. Various types of optic neuropathy, including compressive optic neuropathy and ischemic optic neuropathy, can affect the macula and ganglion cell inner plexiform layer (GCIPL). However, in glaucoma, the inferotemporal region is frequently affected first. The temporal raphe sign is an important sign for distinguishing glaucoma from other neuropathies. The temporal raphe sign is positive if there is a horizontal straight line longer than onehalf of the inner-to-outer-annulus length on the macular GCIPL thickness map. (B) Optovue ONH/GCC OU report of a glaucoma patient. Ganglion cell complex (GCC) significant map shows thinning of inner macula layers in the inferior regions. Tabular data also provides the average GCC, as well as focal loss volume (FLV) and general loss volume (GLV). (C) Heidelberg Posterior Pole Asymmetry Analysis Report: Thickness of whole retina as well as individual layers will be shown on an $8 \times 8$ grid and Hemisphere Asymmetry Map. Thinning of inferotemporal macula is evident in both maps. (D) Topcon Wide Glaucoma report provides GCIPL data (GCL+) or GCC data (GCL++, not shown) in the macula and optic nerve and can be combined with VF results (Hood Glaucoma report). 


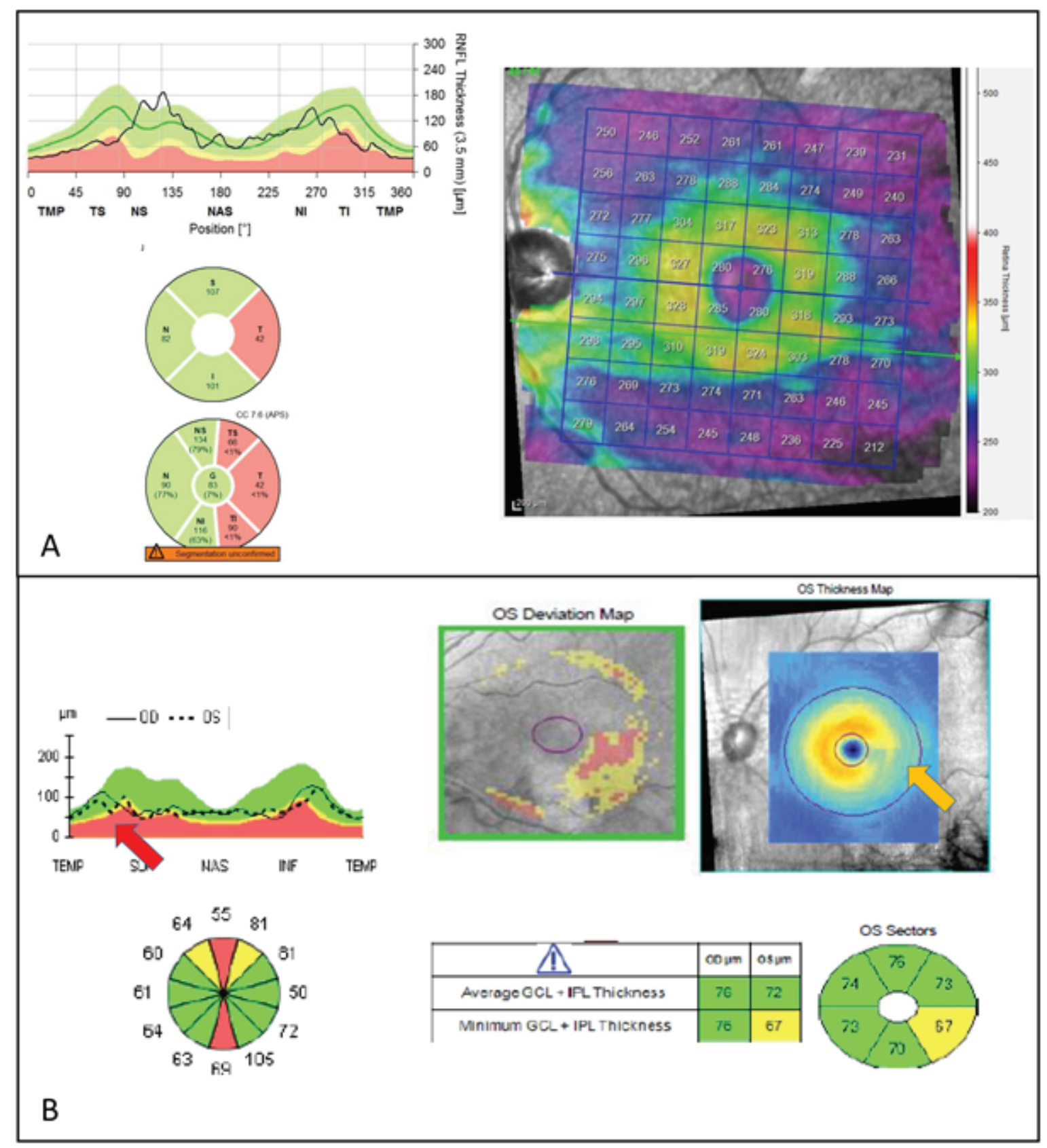

Figure 2. (A) Myopic eye without glaucoma. Myopic eye shows "red disease" with abnormal sectors in the inferotemporal, supratemporal, and temporal sectors which is probably due to temporal displacement of RNFL peaks and not glaucomatous damage. This is confirmed by normal macula thickness in the Posterior Pole Map. (B) Myopic eye with glaucoma. The OCT image shows thinning of the inferior and superior quadrants. This "red disease" might be due to the temporal displacement of RNFL peak. However, GCIPL report shows typical raphe sign (yellow arrow) and inferotemporal macula inner layer thinning suggesting glaucomatous damage. Detailed examination of RNFL profile also depicts a decrease in thickness of superior peak (red arrow).

\section{Spectralis SD-OCT}

Spectralis OCT (Heidelberg Engineering $\mathrm{GmbH}$ ) instrument uses Posterior Pole Analysis (PPA, or Posterior Pole Asymmetry Analysis) algorithm to capture macular images composed of 61 distinct horizontal B-scans (X768 A-scans) that are aligned in parallel to Bruch's membrane opening (BMO)-fovea axis. Each horizontal Bscan is repeated 9-11 times and averaged to decrease speckle noise. The latest software (Glaucoma Module Premium Edition) provides 

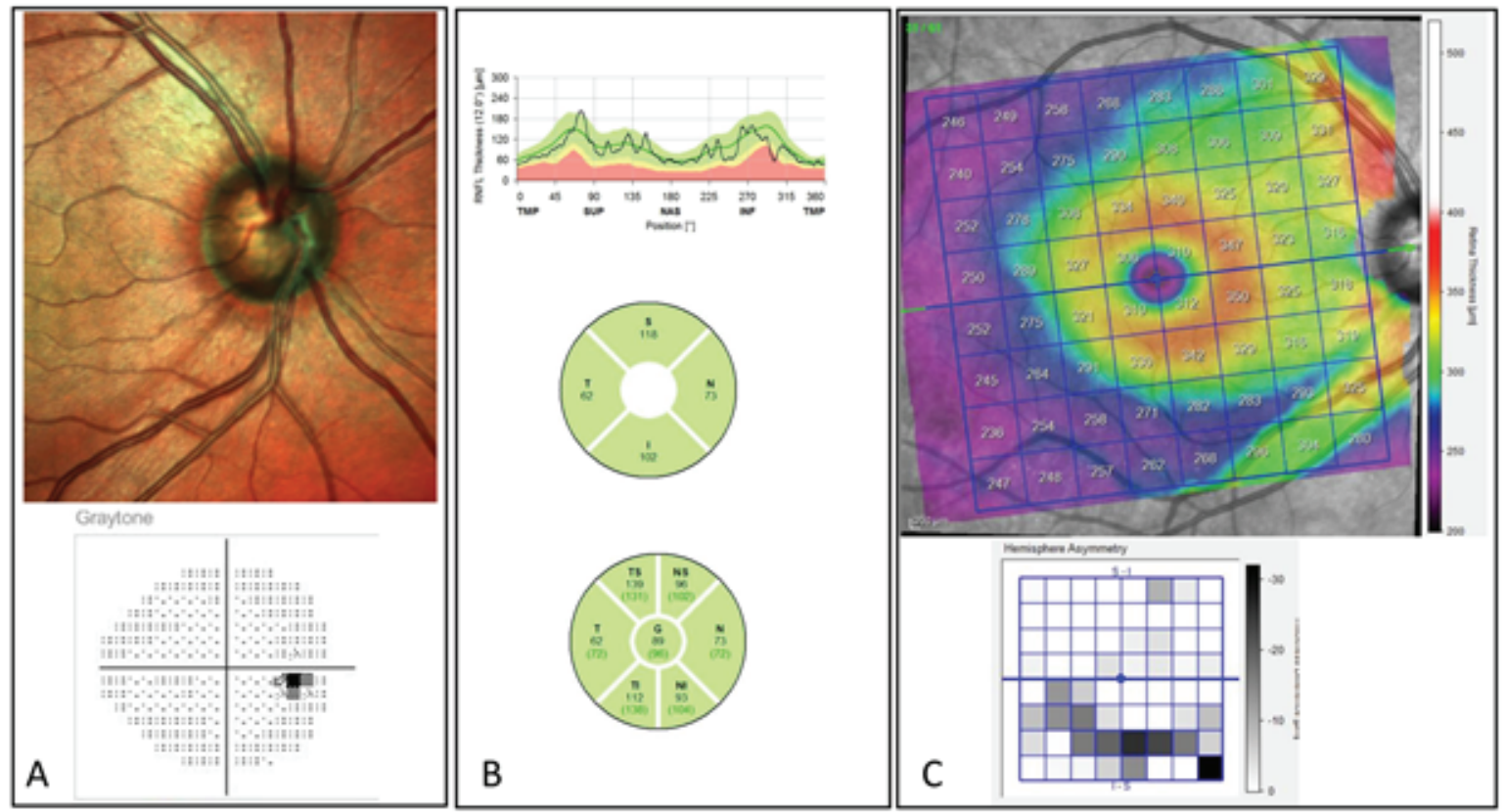

Figure 3. An early glaucoma case with normal VF (A) in which Spectralis RNFL Single report shows normal mean RNFL thickness values and normal sectoral value (B). Posterior Pole Thickness Map and Hemisphere Asymmetry Map shows area of thinning in the macular area corresponded to the location of narrow RNFL defect in optic disc photo.

automated segmentation of the layers of interest. The output displays an $8 \times 8$ thickness grid $(64$ superpixels, $3^{\circ}$ wide) for each layer of interest and enables direct comparison between the superpixels of the fellow eyes as well as the corresponding superior and inferior superpixels of the same eye. Currently, no comparison to the normative data is available on the PPA ${ }^{[18]}$ [Figure $1 \mathrm{C}]$.

\section{Topcon 3D-OCT}

There are multiple Topcon 3D-OCT (Topcon, Inc., Paramus, NJ) instruments of different generations including 3D-OCT 1000, 3D-OCT 2000, and a newer swept-source OCT (DRI OCT-1) device. Topcon 3D-OCT measures a $6 \times 6 \mathrm{~mm}^{2}$ area with a protocol of $128 \times 512$ A-scans/image. DR OCT-1 performs faster measurements and is capable of acquiring wide-field scans over a 12 $\times 9 \mathrm{~mm}^{2}$ area with $256 \times 512$ A-scans/image protocol. In the wide-field report, measurements of mRNFL, GCIPL, and GCC are presented ${ }^{[18]}$ [Figure 1D].

\section{Utility in the Detection of Early Glaucoma}

It is well-known that disease severity affects the diagnostic performance of OCT parameters in glaucoma ${ }^{[22-25]}$ which is mostly represented using the Area Under the Receiver Operating Characteristic curve (AUROC). Hence, attempts have been made to evaluate the diagnostic accuracy of macular OCT parameters and compare their performance to those of $\mathrm{ONH}$ and $\mathrm{cpRNFL}$ thickness at earlier (pre-perimetric and mild perimetric) stages of glaucoma. Their findings reveal excellent and comparable diagnostic performance of macular and cpRNFL parameters in early glaucoma. ${ }^{[2-34]}$ In one of these studies, Kim et al found inferotemporal GCIPL as the macular parameter with the highest diagnostic performance (AUROC $=0.82$ ) comparable to those of best parameters of RNFL (7 o'clock sector, AUROC $=0.76$ ) and $\mathrm{ONH}$ (rim area, AUROC = 0.77). ${ }^{[32]}$ Other studies have reported comparable diagnostic performances of macular GCIPL and cpRNFL deviation maps in the detection of preperimetric glaucoma and good performance of macular GCA maps in the detection of early glaucoma (VF mean deviation [MD] $>-6 \mathrm{~dB}$ ) with the detection rate of up to $87.8 \%$ (in the deviation map). ${ }^{[28,33]}$ Importantly, it is shown that glaucomatous damage to the macular region can happen early in the disease course and sole reliance on a combination of 24-2 VF tests and optic disc OCT can underestimate or even miss the damage. ${ }^{[19]}$ With this respect, Kim and 
colleagues studied a group of 186 glaucoma patients using Cirrus HD-OCT instrument and found that in a subgroup of patients, defects are evident on macular GCIPL but not on cpRNFL deviation maps. However, all cases with a defect on cpRNFL deviation map had a corresponding defect on macular GCIPL deviation map. Based on these findings, they suggested that macular OCT is capable of identifying early glaucomatous damages that may not be apparent on OCT scans of the disc area. ${ }^{[35]}$

Another useful sign for the diagnosis of early glaucoma in macular OCT is the presence of temporal horizontal raphe on GCIPL deviation maps [Figure 1A]. It is defined based on the intuition that early glaucomatous damage preferentially affects one hemifield more than the other. Kim et al developed GCIPL hemifield test which is an automated program for the detection of glaucoma based on this finding. They showed that this test has a very high diagnostic performance for the detection of pre-perimetric glaucoma $(A \cup R O C=$ 0.97 ) and early perimetric glaucoma (AUROC = 0.96). ${ }^{[36]}$ Moreover, in a separate study it was shown that the presence of this sign can be a useful indicator to discriminate glaucomatous from other non-glaucomatous causes of optic neuropathy in eyes with GCIPL thinning. ${ }^{[37]}$ However, these findings from separate groups of Asian glaucoma patients with a presumable high prevalence of normal tension glaucoma may not be generalizable to glaucoma patients of other ethnicities.

The association between distinct glaucomatous optic disc appearance and the presence of central VF defects has been a recent focus of interest. Ekici and collaborators demonstrated that early macular damage in glaucoma patients tends to happen more in optic discs with focal ischemic and myopic phenotypes compared to those with generalized cup enlargement phenotype. ${ }^{[38]}$ Likewise, eyes with optic disc hemorrhage have been associated with more degree of central macular involvement and parafoveal scotoma. In a study by Liu et al, GCIPL showed higher proportional rates of thinning and greater association with functional progression compared to cpRNFL. ${ }^{[39]}$ Accordingly, macular structural evaluation using OCT can be tailored to the individual patients based on the optic disc appearance and this has the potential to enhance the diagnosis and management of early glaucoma.

\section{Monitoring of Advanced Glaucoma}

Monitoring of advanced glaucoma is another challenging task in clinical practice where VF tests show increased variability. Evaluating structural change alongside functional performance can be a useful adjunct if the measurements are reproducible, demonstrate acceptable global and regional structure-function relationship, and fall within the dynamic range of variability. The concept of measurement floor points to another limitation in the evaluation of advanced glaucoma. It is ascribed to a level beyond which the structural measurements no longer show a decremental pattern with worsening of the disease and is believed to represent the thickness of the remining non-neural tissues. Despite that the dynamic range for $c p R N F L$ extends to -8 to $-10 \mathrm{~dB} M D$, macular OCT reaches measurement floor at more advanced glaucomatous damage. ${ }^{[18,40]}$ Bowd and collaborators defined a region of interest approach to estimate the measurement floors of optic disc and macular parameters in a longitudinal cohort of moderate to advanced glaucoma patients (MD $\leq-8 \mathrm{~dB}$ ). They found that the baseline region of interest percentage above the measurement floor for advanced glaucoma cases (MD $\leq-$ $12 \mathrm{~dB}$ ) was highest for GCIPL thickness (36\%), followed by minimum rim width (19\%) and cpRNFL thickness (14\%). As a conclusion, they suggested that macular GCIPL thickness might be a better candidate for monitoring progression in advanced glaucoma compared to optic disc parameters. ${ }^{[41]}$ In another study, Belghith and colleagues found that only GCIPL thickness (compared to cpRNFL) had a significantly faster rate of progression in highly advanced glaucoma patients (MD $<-21$ dB) compared to healthy subjects. ${ }^{[42]}$ Similarly, Lavinsky et al based on an average of four years of follow-up including eyes with a median MD of -10.2 $\mathrm{dB}$ reported an average $-0.57 \mu \mathrm{m} /$ year decline in GCIPL thickness compared to a nonsignificant rate of change for cpRNFL $(0.009 \mu \mathrm{m} /$ year $) .{ }^{[43]}$

In addition, macular OCT measurements have shown high reproducibility in advanced disease, good correlation with VF sensitivity measures, and preserved dynamic range at a stage when the reliability of VF tests decline. A small number of studies have shown that the variability of macular measurements does not significantly increase as the disease gets worse. ${ }^{[4,45]}$ This recommends macular measurements like GCC and GCIPL 
thickness as possible biomarkers for the evaluation of advanced glaucoma; however, it must be kept in mind that accurate segmentation of different macular layers becomes more challenging with worsening of the disease. ${ }^{[18]}$

\section{Utility in the Detection of Glaucoma in Myopic Eyes}

Evaluation of glaucoma in myopic eyes is another area where macular images may yield additional benefit compared to $\mathrm{ONH}$ images. Evaluation and diagnosis of glaucoma in highly myopic eyes is challenging. It has been shown that $\mathrm{ONH}$ parameters have a worse performance in the detection of glaucoma in highly myopic eyes compared to non-highly myopic eyes while GCC's performance remains the same. ${ }^{[46]}$ We know that myopia affects the patterns of RNFL distribution measured by the SD-OCT instruments resulting in temporal displacement of RNFL peaks on thickness plots with possible influence on the diagnostic performance of OCT measurements. ${ }^{[4]}$ Therefore, the so-called "red disease" is not uncommon when clinicians evaluate $\mathrm{ONH}$ repots in these patients [Figure 2A]. This false positive in cpRNFL color code is especially common in inferior quadrants. ${ }^{[48]}$ In addition, the morphology of the $\mathrm{ONH}$ might be altered in highly myopic patients as a result of $\mathrm{ONH}$ tilt and the presence of peripapillary atrophy. These anatomical changes may lead to OCT artifacts $^{[49]}$ and potentially affect the performance of segmentation algorithms especially in the $\mathrm{ONH}$ due to a more complex anatomy compared to the macula. ${ }^{[50,51]}$ In the same line, investigators have shown the superiority of macular over $\mathrm{ONH}$ parameters for the diagnosis of glaucoma in highly myopic eyes; ${ }^{[52-56]}$ although, there are some reports showing a comparable performance between the measurements of these two areas. ${ }^{[29,57-60]}$ Inferotemporal GCIPL thickness is the best macular parameter to detect glaucomatous damage in highly myopic eyes especially at the pre-perimetric stage ${ }^{[57]}$ [Figure 2B]. Kim and colleagues studied Asian high myopic patients and demonstrated an excellent diagnostic accuracy for GCIPL hemifield test to detect glaucomatous damage $($ AUROC $=0.94){ }^{[59]}$ Hence, the presence of temporal horizontal raphe on GCIPL thickness map in high myopic eyes may serve as a useful diagnostic clue to detect glaucoma.

\section{Risk Assessment}

As glaucomatous VF damage is generally irreversible, early intervention is required to prevent further functional deterioration and potential blindness. ${ }^{[61,62]}$ This highlights the role of earlier disease detection and risk stratification of patients according to the future rates of glaucoma progression. Clinical parameters that are associated with prognostic utility in terms of future glaucoma progression are age, the level and fluctuation of intraocular pressure, central corneal thickness, disc hemorrhage, and the diagnosis of pseudoexfoliative glaucoma. ${ }^{[63-71]}$ Certain high risk groups may not only benefit from earlier intervention, but also require closer follow-up appointments and diagnostic tests for the evaluation of disease progression. Macular OCT imaging can be a useful modality in some of these patients. Recently, Shukla and colleagues ${ }^{[72]}$ showed that the presence of disc hemorrhage is associated with more severe damage on 10-2 VF test and faster rate of central VF progression. Hence, this subgroup of patients may benefit from regular macular OCT monitoring for earlier detection of glaucoma progression. Another study showed that the presence of temporal raphe sign on baseline macular GCIPL deviation maps of elderly patients with enlarged vertical cup-todisc ratio is associated with faster progression to normal tension glaucoma. ${ }^{[7]}$ In addition to the aforementioned clinical settings, it has been shown that evaluating macular structure using OCT can further enhance the performance of prognostic models and consequently improve our understanding of glaucoma progression. Anraku and associates evaluated the performance of different baseline structural (macular and cPRNFL OCT) and functional parameters in a cohort of early glaucoma eyes for the detection of future VF progression. The cohort was classified into two groups of slow (MD rate $>-0.4 \mathrm{~dB} /$ year) and fast (MD rate $<-0.4 \mathrm{~dB} /$ year) progressors. Only thinner macular GCC at baseline was a significant predictor of future fast progression. ${ }^{[74]}$ Moreover, in two separate studies, Zhang and colleagues demonstrated that among baseline SD-OCT measurements, GCC focal loss volume (FLV) is the best single predictor for subsequent glaucoma conversion in pre-perimetric glaucoma patients ${ }^{[75]}$ and for VF progression in patients with established glaucoma. ${ }^{[76]}$ In the first study, 
they found that eyes with abnormal or borderline GCC-FLV have a four-fold increase in the risk of future glaucoma conversion over a six-year period. In the second study, they reported that abnormal GCC-FLV at baseline leads to a triple increase in the risk of future VF progression based on an average 3.7 years follow-up of 277 eyes with established glaucoma and average baseline VF $\mathrm{MD}$ of $-4.8 \mathrm{~dB}$. Hou et al evaluated the temporal relationship between progressive GCIPL thinning, cpRNFL thinning, and VF progression in a cohort of patients with primary open-angle glaucoma with a follow-up duration of more than five years. They found that progressive GCIPL and cpRNFL thinning are mutually predictive and both are indicative of VF progression. ${ }^{[77]}$ They suggested that integrating macular and cpRNFL parameters may probably lead to earlier detection of disease progression in glaucoma patients.

\section{Applications of Artificial Intelligence (Al)}

In the recent years, the applications of $\mathrm{Al}$ in general (and deep learning networks in particular) into medicine has led to the introduction of numerous automated diagnostic modalities. Al techniques have many implications in machine vision tasks including image classification with the performance sometimes higher than that of humans $^{[78]}$ and unsupervised identification of different patterns that exist in large datasets of images. A widespread use of different imaging modalities in ophthalmology research and clinical practice makes this medical subspecialty a major area for the implementation of these novel algorithms to assist in diagnosis and improve the currently used image analysis techniques. ${ }^{[79]}$ A great proportion of publications on $\mathrm{Al}$ methods in glaucoma have focused on the detection of the disease using different inputs like fundus and OCT images. Asaoka and colleagues developed and validated a deep learning model to accurately (AUROC $=93.7 \%)$ detect early open-angle glaucoma (MD $>-5 \mathrm{~dB}$ ) using macular OCT information. ${ }^{[80]}$ Another recent study developed a $3 D$ deep learning system to detect patients that need to be referred to a glaucoma specialist based on the volumetric macular OCT information. The overall accuracy of their proposed surveillance system was high (AUROC $=0.88$ ) with a relatively well preserved performance among eyes with different degrees of myopia. ${ }^{[81]}$ Development of deep learning models to evaluate the details of structure-function relationship has been another focus of Al investigations in glaucoma. These models have shown a high accuracy to extract and use the relevant information obtained from macular volumetric OCT scans and provide a corresponding simulation of central VF in glaucoma patients. ${ }^{[82-84]}$ Moreover, Nouri-Mahdavi et al in a recent study showed that VF progression in moderate to advance glaucoma can be partly predicted using combined OCT measurements of peripapillary and macular areas. Of note, they developed and compared separate models using macular or peripapillary measurements and showed that macular models performed better than peripapillary models to detect VF progression. This finding highlights the potential of macular OCT in monitoring patients with moderate to advanced glaucoma. ${ }^{[85]}$ Hopefully, by further refining these Al approaches, automated precise systems for the detection and monitoring of disease progression in glaucoma will become available in the future.

\section{What We Should Do as Clinicians}

While it is common clinical practice to obtain an OCT scan of the disc, many clinicians do not routinely obtain a scan of the macular region for patients with glaucoma or suspected glaucoma. As previously discussed, macula SD-OCT has been helpful for the earlier detection of glaucoma particularly in eyes with certain $\mathrm{ONH}$ phenotypes, eyes with $\mathrm{DH}$, and myopic eyes. For example, Figure 3 shows a case in which the cpRNFL report appears normal, while the macula scan shows apparent GCIPL thinning. Macula scans may also serve as a clue for clinicians for the presence of parafoveal scotoma, which should receive attention and be further evaluated with a 10-2 VF test. In addition, macula scans play an important role in monitoring eyes with advanced disease, as they may help clinicians identify disease progression and decide to escalate treatment. Consequently, one may ask, "When do you perform an OCT scan of the macula?" Although macula scans can be selectively ordered for those patients who may be most likely to benefit from it (as discussed earlier), obtaining OCT scans is so efficient today that many experts recommend routinely performing both disc and macula scans for all patients with glaucoma and suspected glaucoma, thus have 
a comprehensive glaucoma assessment of the patients.

\section{Limitations}

Like every other imaging modality, there are some limitations in the use macular OCT for glaucoma practice and research that clinicians need to consider. First, GCIPL analysis with the SDOCT may be complicated by coexisting macular pathology and scan artifacts. Most currently available data on macular OCT in glaucoma are obtained from studies that have excluded eyes with other macular pathologies and also poor quality images like those with artifacts and lower signal strength. Thus, one needs to expect higher variability of macular measurements in real world scenarios. The presence of age-related macular pathologies and drusen may disrupt the correct segmentation of the macular layers that are important in the diagnosis and monitoring of glaucoma patients especially in the elderly. Recent studies using GCIPL analysis have excluded up to $6 \%$ of scans due to machine segmentation or acquisition error. ${ }^{[86]}$ Moreover, any retinal diseases involving macular areas such as the epiretinal membrane, age-related macular degeneration, or macular edema can affect macular GCIPL thickness and reducing performance of the macula scans for detection of glaucoma. Similarly, macula scans are not helpful for detection of glaucoma in eyes with myopic myopathy. Even in eyes without maculopathy, abnormal diagnostic classifications on GCIPL map can be seen in up to $40 \%$ of myopic eyes with diffuse, circular pattern being the predominant form. ${ }^{[87]}$ In addition, macular measurements obtained by different OCT devices are not interchangeable despite showing a fair degree of correlation. ${ }^{[88]}$ Finally, it has to be mentioned that glaucomatous damage with a high angular distance from the BMO-fovea axis may fall out of the measurement territory of macular OCT images depending on the OCT instrument and software and consequently not be identifiable on the deviation maps. Clinicians need to be aware of this issue and take it into consideration. ${ }^{[28,32]}$

\section{CONCLUSION}

Macular OCT is a useful imaging modality in glaucoma management and research. It provides complementary information to the conventionally used modalities by glaucoma specialists especially in the evaluation of patients with early macular damage and/or high myopia and monitoring of the advanced disease. With the development and widespread use of $\mathrm{Al}$ techniques in medicine, macular OCT information can be integrated with the information obtained from optic disc OCT and VF assessment to provide a more comprehensive picture of the true nature of glaucomatous damage and progression. This will definitely enhance the quality of medical care and research in the future.

\section{Financial Support and Sponsorship}

This study was supported by the UC Tobacco Related Disease Research Program Grant T31IP1511.

\section{Conflicts of Interest}

There are no conflicts of interest.

\section{REFERENCES}

1. Weinreb RN, Leung CK, Crowston JG, Medeiros FA, Friedman DS, Wiggs $J L$, et al. Primary open-angle glaucoma. Nat Rev Dis Primers 2016;2:1-19.

2. Weinreb RN, Aung T, Medeiros FAJJ. The pathophysiology and treatment of glaucoma: a review. JAMA 2014;311:19011911.

3. Quigley HA, Broman AT. The number of people with glaucoma worldwide in 2010 and 2020. Br J Ophthalmol 2006;90:262-267.

4. Quigley HA, Dunkelberger GR, Green WR. Retinal ganglion cell atrophy correlated with automated perimetry in human eyes with glaucoma. Am J Ophthalmol 1989;107:453-464.

5. Quigley HA, Nickells RW, Kerrigan LA, et al. Retinal ganglion cell death in experimental glaucoma and after axotomy occurs by apoptosis. Invest Ophthalmol Vis Sci 1995;36:774-786.

6. Coleman AL. Glaucoma. The Lancet 1999;354:1803-1810.

7. Weinreb RN, Friedman DS, Fechtner RD, Cioffi GA, Coleman AL, Girkin CA, et al. Risk assessment in the management of patients with ocular hypertension. $A m \mathrm{~J}$ Ophthalmol 2004;138:458-467.

8. Alencar LM, Zangwill LM, Weinreb RN, Bowd C, Vizzeri G, Sample PA, et al. Agreement for detecting glaucoma progression with the GDx guided progression analysis, automated perimetry, and optic disc photography. Opthalmology 2010;117:462-470.

9. Alexandrescu C, Dascalu A, Panca A, Sescioreanu A, Mitulescu C, Ciuluvica R, et al. Confocal scanning laser ophthalmoscopy in glaucoma diagnosis and management. J Med Life 2010;3:229. 
10. Andreou PA, Wickremasinghe SS, Asaria RH, Tay E, Franks WA. A comparison of HRT II and GDx imaging for glaucoma detection in a primary care eye clinic setting. Eye 2007;21:1050-1055.

11. Belghith $\mathrm{A}$, Balasubramanian $\mathrm{M}$, Bowd $\mathrm{C}$, Weinreb RN, Zangwill LM. A unified framework for glaucoma progression detection using Heidelberg Retina Tomograph images. Comput Med Imaging Graph 2014;38:411-420.

12. Haleem MS, Han L, van Hemert J, Fleming A, Pasquale LR, Silva PS, et al. Regional image features model for automatic classification between normal and glaucoma in fundus and scanning laser ophthalmoscopy (SLO) images. J Med Syst 2016;40:132.

13. Lin SC, Singh K, Jampel HD, Hodapp EA, Smith SD, Francis BA, et al. Optic nerve head and retinal nerve fiber layer analysis: a report by the American Academy of Ophthalmology. Ophthalmology 2007;114:1937-1949.

14. Sharma P, Sample PA, Zangwill LM, Schuman JS. Diagnostic tools for glaucoma detection and management. Surv Ophthalmol 2008;53:S17-S32.

15. Stein JD, Talwar N, LaVerne AM, Nan B, Lichter PR. Trends in use of ancillary glaucoma tests for patients with open-angle glaucoma from 2001 to 2009. Ophthalmology 2012;119:748-758.

16. Drexler W, Fujimoto JG. State-of-the-art retinal optical coherence tomography. Prog Retin Eye Res 2008;27:4588.

17. Schuman JS, Hee MR, Arya AV, Pedut-Kloizman T, Puliafito CA, Fujimoto JG, et al. Optical coherence tomography: a new tool for glaucoma diagnosis. Curr Opin Ophthalmol 1995;6:89-95.

18. Mohammadzadeh V, Fatehi N, Yarmohammadi A, Woong Lee J, Sharifipour F, Daneshvar R, et al. Macular imaging with optical coherence tomography in glaucoma. Surv Ophthalmol 2020;65:597-638.

19. Hood DC, De Moraes CG. Challenges to the common clinical paradigm for diagnosis of glaucomatous damage with OCT and visual fields. Invest Ophthalmol Vis Sci 2018;59:788-791.

20. Hood DC, De Moraes CG. Four questions for every clinician diagnosing and monitoring glaucoma. $J$ Glaucoma 2018;27:657.

21. Hood DC, Raza AS, de Moraes CGV, Liebmann JM, Ritch RJPir. Glaucomatous damage of the macula. Prog Retin Eye Res 2013;32:1-21.

22. Zangwill LM, Jain S, Racette L, Ernstrom KB, Bowd C, Medeiros FA, et al. The effect of disc size and severity of disease on the diagnostic accuracy of the Heidelberg Retina Tomograph Glaucoma Probability Score. Invest Ophthalmol Vis Sci 2007;48:2653-2660.

23. Rao HL, Leite MT, Weinreb RN, Zangwill LM, Alencar LM, Sample PA, et al. Effect of disease severity and optic disc size on diagnostic accuracy of RTVue spectral domain optical coherence tomograph in glaucoma. Invest Ophthalmol Vis Sci 2011;52:1290-1296.

24. Medeiros FA, Zangwill LM, Bowd C, Sample PA, Weinreb $\mathrm{RN}$. Influence of disease severity and optic disc size on the diagnostic performance of imaging instruments in glaucoma. Invest Ophthalmol Vis Sci 2006;47:1008-1015.

25. Leite MT, Zangwill LM, Weinreb RN, Rao HL, Alencar LM, Sample PA, et al. Effect of disease severity on the performance of cirrus spectral-domain oct for glaucoma diagnosis. Invest Ophthalmol Vis Sci 2010;51:4104-4109.

26. Nouri-Mahdavi K, Nowroozizadeh S, Nassiri N, Cirineo N, Knipping S, Giaconi J, et al. Macular ganglion cell/inner plexiform layer measurements by spectral domain optical coherence tomography for detection of early glaucoma and comparison to retinal nerve fiber layer measurements. Am J Ophthalmol 2013;156:1297-1307.e2.

27. Shin H-Y, Park H-YL, Jung Y, Choi J-A, Park CKJO. Glaucoma diagnostic accuracy of optical coherence tomography parameters in early glaucoma with different types of optic disc damage. Ophthalmology 2014;121:1990-1997.

28. Hwang YH, Jeong YC, Kim HK, Sohn YHJO. Macular ganglion cell analysis for early detection of glaucoma. Ophthalmology 2014;121:1508-1515.

29. Jeoung JW, Choi YJ, Park KH, Kim DM. Macular ganglion cell imaging study: glaucoma diagnostic accuracy of spectral-domain optical coherence tomography. Invest Ophthalmol Vis Sci 2013;54:4422-4429.

30. Mwanza J-C, Durbin MK, Budenz DL, Sayyad FE, Chang RT, Neelakantan A, et al. Glaucoma diagnostic accuracy of ganglion cell-inner plexiform layer thickness: comparison with nerve fiber layer and optic nerve head. Ophthalmology 2012;119:1151-1158.

31. Shin H-Y, Park H-YL, Jung K-I, Choi J-A, Park CK. Glaucoma diagnostic ability of ganglion cell-inner plexiform layer thickness differs according to the location of visual field loss. Ophthalmology 2014;121:93-99.

32. Kim MJ, Jeoung JW, Park KH, Choi YJ, Kim DM. Topographic profiles of retinal nerve fiber layer defects affect the diagnostic performance of macular scans in preperimetric glaucoma. Invest Ophthalmol Vis Sci 2014;55:2079-2087.

33. Kim MJ, Park KH, Yoo BW, Jeoung JW, Kim HC, Kim DM. Comparison of macular GCIPL and peripapillary RNFL deviation maps for detection of glaucomatous eye with localized RNFL defect. Acta Ophthalmol 2015;93:e22e28.

34. Sung M-S, Yoon J-H, Park S-W. Diagnostic validity of macular ganglion cell-inner plexiform layer thickness deviation map algorithm using cirrus HD-OCT in preperimetric and early glaucoma. J Glaucoma 2014;23:e144-e151.

35. Kim YK, Jeoung JW, Park KH. Inferior macular damage in glaucoma: its relationship to retinal nerve fiber layer defect in macular vulnerability zone. J Glaucoma 2017;26:126132.

36. Kim YK, Yoo BW, Kim HC, Park KH. Automated detection of hemifield difference across horizontal raphe on ganglion cell-inner plexiform layer thickness map. Ophthalmology 2015;122:2252-2260.

37. Lee J, Kim YK, Ha A, Kim YW, Baek SU, Kim J-S, et al. Temporal raphe sign for discrimination of glaucoma from optic neuropathy in eyes with macular ganglion cell-inner plexiform layer thinning. Ophthalmology 2019;126:11311139.

38. Ekici E, Moghimi S, Hou H, Proudfoot J, Zangwill LM, Do JL, et al. Central visual field defects in patients with distinct glaucomatous optic disc phenotypes. Am J Ophthalmol 2021;223:229-240. 
39. Liu X, Lau A, Hou H, Moghimi S, Proudfoot JA, Chan E, et al. Progressive thinning of retinal nerve fiber layer and ganglion cell-inner plexiform layer in glaucoma eyes with disc hemorrhage. Ophthalmol Glaucoma 2021:S2589S4196(21)00031-4.

40. Miraftabi A, Amini N, Morales E, Henry S, Yu F, Afifi A, et al. Macular SD-OCT outcome measures: comparison of local structure-function relationships and dynamic range. Invest Ophthalmol Vis Sci 2016;57:4815-4823.

41. Bowd C, Zangwill LM, Weinreb RN, Medeiros FA, Belghith A. Estimating optical coherence tomography structural measurement floors to improve detection of progression in advanced glaucoma. Am J Ophthalmol 2017;175:37-44.

42. Belghith A, Medeiros FA, Bowd C, Liebmann JM, Girkin CA, Weinreb RN, et al. Structural change can be detected in advanced-glaucoma eyes. Invest Ophthalmol Vis Sci 2016;57:OCT511-OCT518.

43. Lavinsky F, Wu M, Schuman JS, Lucy KA, Liu M, Song $Y$, et al. Can macula and optic nerve head parameters detect glaucoma progression in eyes with advanced circumpapillary retinal nerve fiber layer damage? Ophthalmology 2018;125:1907-1912.

44. Miraftabi A, Amini N, Gornbein J, Henry S, Romero P, Coleman $\mathrm{AL}$, et al. Local variability of macular thickness measurements with SD-OCT and influencing factors. Trans/ Vis Sci Technol 2016;5:5-5.

45. Pearce JG, Maddess T. Inter-visit test-retest variability of OCT in glaucoma. Optom Vis Sci 2017;94:404-410.

46. Akashi A, Kanamori A, Ueda K, Inoue $Y$, Yamada $Y$, Nakamura M. The ability of SD-OCT to differentiate early glaucoma with high myopia from highly myopic controls and nonhighly myopic controls. Invest Ophthalmol Vis Sci 2015;56:6573-6580.

47. Kang SH, Hong SW, Im SK, Lee SH, Ahn MD. Effect of myopia on the thickness of the retinal nerve fiber layer measured by Cirrus HD optical coherence tomography. Invest Ophthalmol Vis Sci 2010;51:4075-4083.

48. Kim NR, Lim H, Kim JH, Rho SS, Seong GJ, Kim CY. Factors associated with false positives in retinal nerve fiber layer color codes from spectral-domain optical coherence tomography. Ophthalmology 2011;118:1774-1781.

49. Akman A, Bayer A, Nouri-Mahdavi K. Optical coherence tomography in glaucoma: a practical guide. Springer, 2018.

50. Kim KE, Park KH. Macular imaging by optical coherence tomography in the diagnosis and management of glaucoma. Br J Ophthalmol 2018;102:718-724.

51. Lee M-W, Park K-S, Lim H-B, Jo Y-J, Kim J-Y. Long-term reproducibility of GC-IPL thickness measurements using spectral domain optical coherence tomography in eyes with high myopia. Sci Rep 2018;8:1-8.

52. Nakano N, Hangai M, Noma H, Nukada M, Mori S, Morooka $\mathrm{S}$, et al. Macular imaging in highly myopic eyes with and without glaucoma. Am J Ophthalmol 2013;156:511-523.e6.

53. Shoji T, Nagaoka $Y$, Sato H, Chihara E. Impact of high myopia on the performance of SD-OCT parameters to detect glaucoma. Graefes Arch Clin Exp Ophthalmol 2012;250:1843-1849.

54. Shoji T, Sato H, Ishida M, Takeuchi M, Chihara E. Assessment of Glaucomatous changes in subjects with high myopia using spectral domain optical coherence tomography. Invest Ophthalmol Vis Sci 2011;52:10981102.
55. Wang W-W, Wang H-Z, Liu J-R, Zhang X-F, Li M, Huo Y-J, et al. Diagnostic ability of ganglion cell complex thickness to detect glaucoma in high myopia eyes by Fourier domain optical coherence tomography. Int J Ophthalmol 2018;11:791.

56. Zhang $\mathrm{Y}$, Wen $\mathrm{W}$, Sun $\mathrm{X}$. Comparison of several parameters in two optical coherence tomography systems for detecting glaucomatous defects in high myopia. Invest Ophthalmol Vis Sci 2016;57:4910-4915.

57. Seol BR, Jeoung JW, Park KH. Glaucoma detection ability of macular ganglion cell-inner plexiform layer thickness in myopic preperimetric glaucoma. Invest Ophthalmo/ Vis Sci 2015;56:8306-8313.

58. Choi YJ, Jeoung JW, Park KH, Kim DM. Glaucoma detection ability of ganglion cell-inner plexiform layer thickness by spectral-domain optical coherence tomography in high myopia. Invest Ophthalmol Vis Sci 2013;54:2296-2304.

59. Kim YK, Yoo BW, Jeoung JW, Kim HC, Kim HJ, Park $\mathrm{KH}$. Glaucoma-diagnostic ability of ganglion cell-inner plexiform layer thickness difference across temporal raphe in highly myopic eyes. Invest Ophthalmol Vis Sci 2016;57:5856-5863.

60. Akashi A, Kanamori A, Ueda K, Inoue Y, Yamada Y, Nakamura M. The ability of SD-OCT to differentiate early glaucoma with high myopia from highly myopic controls and nonhighly myopic controls. Invest Ophthalmol Vis Sci 2015;56:6573-6580.

61. Caprioli J. The importance of rates in glaucoma. Am J Ophthalmol 2008;145:191-192.

62. Hernández R, Burr J, Vale L, Azuara-Blanco A, Cook JA, Banister K. Monitoring ocular hypertension, how much and how often? A cost-effectiveness perspective. $\mathrm{Br} J$ Ophthalmol 2016;100:1263-1268.

63. Caprioli J, Coleman AL. Intraocular pressure fluctuation: a risk factor for visual field progression at low intraocular pressures in the Advanced Glaucoma Intervention Study. Ophthalmology 2008;115:1123-1129.e3.

64. Gardiner SK, Johnson CA, Demirel S. Factors predicting the rate of functional progression in early and suspected glaucoma. Invest Ophthalmol Vis Sci 2012;53:3598-3604.

65. Jiang X, Varma R, Wu S, Torres M, Azen SP, Francis BA, et al. Baseline Risk factors that predict the development of open-angle glaucoma in a population: the Los Angeles Latino Eye Study. Ophthalmology 2012;119:2245-2253.

66. Leske MC, Heijl A, Hussein M, Bengtsson B, Hyman L, Komaroff $\mathrm{E}$, et al. Factors for glaucoma progression and the effect of treatment: the early manifest glaucoma trial. Arch Ophthalmol 2003;121:48-56.

67. Leske MC, Heijl A, Hyman L, Bengtsson B, Dong L, Yang Z. Predictors of long-term progression in the early manifest glaucoma trial. Ophthalmology 2007;114:1965-1972.

68. Malihi M, Moura Filho ER, Hodge DO, Sit AJ. Long-term trends in glaucoma-related blindness in Olmsted County, Minnesota. Ophthalmology 2014;121:134-141.

69. Medeiros FA, Sample PA, Weinreb RN. Corneal thickness measurements and frequency doubling technology perimetry abnormalities in ocular hypertensive eyes. Ophthalmology 2003;110:1903-1908.

70. Nouri-Mahdavi K, Hoffman D, Coleman AL, Liu G, Li G, Gaasterland D, et al. Predictive factors for glaucomatous 
visual field progression in the Advanced Glaucoma Intervention Study. Ophthalmology 2004;111:1627-1635.

71. Sung KR, Cho JW, Lee S, Yun S-C, Choi J, Na JH, et al. Characteristics of visual field progression in medically treated normal-tension glaucoma patients with unstable ocular perfusion pressure. Invest Ophthalmol Vis Sci 2011;52:737-743.

72. Shukla AG, Sirinek PE, De Moraes CG, Blumberg DM, Cioffi GA, Skaat A, et al. Disc hemorrhages are associated with the presence and progression of glaucomatous central visual field defects. J Glaucoma 2020;29:429-434.

73. Ha A, Kim YK, Kim J-S, Jeoung JW, Park KH. Temporal raphe sign in elderly patients with large optic disc cupping: its evaluation as a predictive factor for glaucoma conversion. Am J Opthalmol 2020;219:205-214.

74. Anraku A, Enomoto N, Takeyama A, Ito H, Tomita G. Baseline thickness of macular ganglion cell complex predicts progression of visual field loss. Graefes Arch Clin Exp Ophthalmol 2014;252:109-115.

75. Zhang X, Loewen N, Tan O, Greenfield DS, Schuman JS, Varma $\mathrm{R}$, et al. Predicting development of glaucomatous visual field conversion using baseline fourier-domain optical coherence tomography. $A m \mathrm{~J}$ Opthalmol 2016;163:29-37.

76. Zhang X, Dastiridou A, Francis BA, Tan O, Varma R, Greenfield DS, et al. Baseline fourier-domain optical coherence tomography structural risk factors for visual field progression in the advanced imaging for glaucoma study. Am J Opthalmol 2016;172:94-103.

77. Hou HW, Lin C, Leung CK-S. Integrating macular ganglion cell inner plexiform layer and parapapillary retinal nerve fiber layer measurements to detect glaucoma progression. Ophthalmology 2018;125:822-831.

78. He K, Zhang X, Ren S, Sun J. Delving deep into rectifiers: surpassing human-level performance on imagenet classification. Proc IEEE Int Conf Comput Vis, 2015:10261034.

79. Medeiros FA. Deep learning in glaucoma: progress, but still lots to do. The Lancet Digital Health 2019;1:e151-e152.
80. Asaoka R, Murata H, Hirasawa K, Fujino Y, Matsuura M, Miki $A$, et al. Using deep learning and transfer learning to accurately diagnose early-onset glaucoma from macular optical coherence tomography images. Am J Ophthalmol 2019;198:136-145.

81. Russakoff DB, Mannil SS, Oakley JD, Ran Ran A, Cheung CY, Dasari S, et al. A 3D deep learning system for detecting referable glaucoma using full OCT macular cube scans. Trans/ Vis Sci Technol 2020;9:12.

82. Hashimoto $\mathrm{Y}$, Asaoka R, Kiwaki T, Sugiura H, Asano S, Murata $\mathrm{H}$, et al. Deep learning model to predict visual field in central $10^{\circ}$ from optical coherence tomography measurement in glaucoma. Br J Ophthalmol 2020;4:507513.

83. Xu L, Asaoka R, Kiwaki T, Murata H, Fujino Y, Matsuura $\mathrm{M}$, et al. Predicting the glaucomatous central 10-degree visual field from optical coherence tomography using deep learning and tensor regression. Am J Ophthalmol 2020;218:304-313

84. Asano S, Asaoka R, Murata H, Hashimoto Y, Miki A, Mori $\mathrm{K}$, et al. Predicting the central 10 degrees visual field in glaucoma by applying a deep learning algorithm to optical coherence tomography images. Sci Rep 2021;11:1-10.

85. Nouri-Mahdavi K, Mohammadzadeh V, Rabiolo A, Edalati K, Caprioli J, Yousefi S. Prediction of visual field progression from OCT structural measures in moderate to advanced glaucoma. Am J Ophthalmol 2021;226:172-181.

86. Awadalla MS, Fitzgerald J, Andrew NH, Zhou T, Marshall $H$, Qassim A, et al. Prevalence and type of artefact with spectral domain optical coherence tomography macular ganglion cell imaging in glaucoma surveillance. PLoS One 2018;13:e0206684.

87. Kim KE, Jeoung JW, Park KH, Kim DM, Kim SH. Diagnostic classification of macular ganglion cell and retinal nerve fiber layer analysis: differentiation of false-positives from glaucoma. Ophthalmology 2015;122:502-510.

88. Mahmoudinezhad G, Mohammadzadeh V, Amini N, Toriz V, Pourhomayoun M, Heydarzadeh S, et al. Local Macular Thickness Relationships between 2 OCT Devices. 2021;4:209-215. 